\begin{tabular}{|c|c|c|c|c|c|c|}
\hline \multirow{4}{*}{ Impact Factor: } & ISRA (India) & $=3.117$ & SIS (USA) & $=0.912$ & ICV (Poland) & $=6.630$ \\
\hline & ISI (Dubai, UAE & $=0.829$ & РИНЦ (Russia & $=0.156$ & PIF (India) & $=1.940$ \\
\hline & GIF (Australia) & $=0.564$ & ESJI (KZ) & $=8.716$ & IBI (India) & $=4.260$ \\
\hline & JIF & $=1.500$ & SJIF (Morocco & $=\mathbf{5 . 6 6 7}$ & OAJI (USA) & $=0.350$ \\
\hline
\end{tabular}

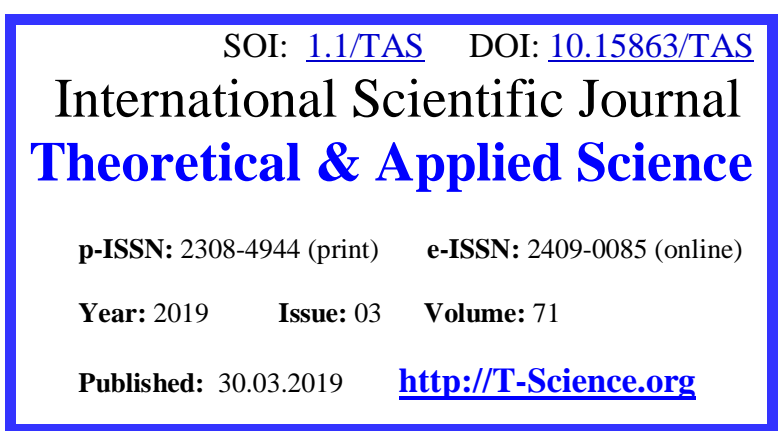

SECTION 7. Mechanics and machine construction.
QR - Issue

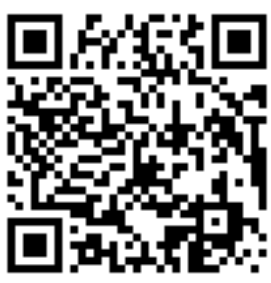

Mc. Eng. Corresponding Member of International Academy of Theoretical and Applied Sciences, Lecturer of Vladimir Industrial College, Russian Federation https://orcid.org/0000-0002-2747-552X chemezov-da@yandex.ru

Irina Pavluhina Lecturer of Vladimir Industrial College, Russian Federation

Alexandra Strunina Lecturer of Vladimir Industrial College,

Russian Federation

Andrey Pesenko Student of Vladimir Industrial College,

Russian Federation

Polina Nikitina

Lecturer of Vladimir Industrial College,

Russian Federation

Tatyana Komarova Lecturer of Vladimir Industrial College,

Russian Federation

Elena Kiseleva Master of Industrial Training, Vladimir Industrial College, Russian Federation

Vadim Maximov Student of Vladimir Industrial College, Russian Federation

\title{
DEFORMATIONS ISOSURFACES OF CANTILEVER AND DOUBLY SUPPORTED STEEL I-BEAMS AT BENDING
}

Abstract: The calculation of deformed state of cantilever and doubly supported steel I-beams after removing of concentrated or distributed loads is presented in the article. Isosurfaces of deformation tensor of the I-beams material for all components are calculated. The conclusion about direction and intensity of volumetric deformation of the Ibeams material by the color isosurfaces is given.

Key words: an I-beam, deformation, concentrated and distributed forces, moment, tensor, a component.

Language: Russian English

Citation: Chemezov, D., et al. (2019). Deformations isosurfaces of cantilever and doubly supported steel Ibeams at bending. ISJ Theoretical \& Applied Science, 03 (71), 501-513.

Soi: http://s-o-i.org/1.1/TAS-03-71-47 Doi: crossef https://dx.doi.org/10.15863/TAS.2019.03.71.47 


\begin{tabular}{|c|c|c|c|c|c|c|}
\hline \multirow{4}{*}{ Impact Factor: } & ISRA (India) & $=\mathbf{3 . 1 1 7}$ & SIS (USA) & $=0.912$ & ICV (Poland) & $=6.630$ \\
\hline & ISI (Dubai, UAE & $=0.829$ & РИНЦ (Russia & $=0.156$ & PIF (India) & $=1.940$ \\
\hline & GIF (Australia) & $=0.564$ & ESJI (KZ) & $=8.716$ & IBI (India) & $=4.260$ \\
\hline & JIF & $=1.500$ & SJIF (Morocco & $=5.667$ & OAJI (USA) & $=0.350$ \\
\hline
\end{tabular}

\section{Introduction}

Description of stress-strain state of metal Ibeams is presented in the works [1-10].

Applied external loads lead to bending of the Ibeams and versatile volumetric deformation in material. Rotation of the I-beam at a certain angle occurs during bending. This process leads to displacement of material layers of the I-beam throughout a volume. Deformations of tensile or compressive that reduce strength of material occur in the inner layers of the I-beam.

Problems solving in the discipline "Technical mechanics" allows to get an overall assessment of stress state of the I-beams. Volumetric plastic deformation of the I-beam material is determined by deformation tensor. A three-dimensional view of deformation of the beam material can be obtained in a short period of time by finite element modeling.

Recommendations for a choice of cross sectional dimensions of the cantilever and doubly supported Ibeams can be formulated in accordance with the conclusion about a degree of plastic deformation of material.

\section{Materials and methods}

Solid models of the I-beams were subjected to loads that have been applied in the COMSOL Multiphysics software environment. Deformation of the cantilever and doubly supported I-beams was calculated. Concentrated and distributed forces, concentrated moment were applied. The loading schemes of the models of the cantilever and doubly supported I-beams are presented in the table 1.

The calculation of the cantilever and doubly supported I-beams was carried out taking into account the reliability factor in relation to loading by the value of 1.2 and the own weight of the beam.

Structural steel has been adopted as material of the I-beam models. Young's modulus and calculated resistance of the beams material were adopted by the values of 206000 and $230 \mathrm{MPa}$, respectively.

The main characteristics of the I-beams:

- the weight of one running meter of the beam $q$ $-8.1 \mathrm{~kg} / \mathrm{m}$;

- inertia moment $J_{x}-171 \mathrm{~cm}^{4}$;

- moment of resistance $W_{x}-34.2 \mathrm{~cm}^{3}$;

- static moment of a half-section $S_{x}-19.7 \mathrm{~cm}^{3}$;

- a web thickness $t-4.1 \mathrm{~mm}$

\section{Results and discussion}

The isosurfaces of rotation of deformation tensor on the solid models of the loaded I-beams are presented in the Fig. $1-9$.

The $x X, y Y$ and $z Z$ components of deformation tensor of material of the cantilever and doubly supported I-beams are zero, i.e. deformation is not observed. The isosurfaces of material deformation of the I-beams mostly have the negative values. This suggests that the beam material is dominated by compressive deformation.

The values of the deformation coefficients were determined for two components ( $y Z$ and $z Y$ ) of deformation tensor of the cantilever I-beams. The deformation coefficients of material have the negative values under the action of concentrated moment clockwise and concentrated force on a loose end of the I-beam. The deformation coefficients of material have the positive values under the action of concentrated force at $1 / 2$ of a span length and distributed force at the entire span length of the I-beam. The positive and negative values of the deformation coefficients of material are observed in other components.

Maximum deformation of compressive and tensile of material of the cantilever I-beam (the loading scheme No. 2) was noted in the planes $z Y$ ($\left.7.53 \cdot 10^{-3}\right)$ and $y Z\left(7.53 \cdot 10^{-3}\right)$, respectively.

Bending leads to deflection of the cantilever and doubly supported I-beams. The deflection value of the I-beams characterizes strength and rigidity of material of a construction element. The values of maximum deflections of the cantilever and doubly supported Ibeams are presented in the summary table 2 .

Maximum deflection of the beam was determined for the second loading scheme (the cantilever I-beam), minimum deflection was determined for the ninth loading scheme (the doubly supported I-beam).

\section{Conclusion}

The layers of material which are oriented along the length and the height of the cantilever I-beam are subjected to maximum deformation.

Loading of the I-beam according to the second scheme leads to maximum deflection which is $1 / 175$ from the maximum span of the beam.

The additional support (at the considered span length) gives rigidity increasing of the I-beam by about 6 times.

Table 1. The loading schemes of the cantilever and doubly supported I-beams. 


\begin{tabular}{|c|c|c|c|c|c|c|}
\hline \multirow{4}{*}{ Impact Factor: } & ISRA (India) & $=3.117$ & SIS (USA) & $=0.912$ & ICV (Poland) & $=6.630$ \\
\hline & ISI (Dubai, UAE & $=0.829$ & РИНЦ (Russia & $=0.156$ & PIF (India) & $=1.940$ \\
\hline & GIF (Australia) & $=0.564$ & ESJI (KZ) & $=8.716$ & IBI (India) & $=4.260$ \\
\hline & JIF & $=1.500$ & SJIF (Morocce & $=5.667$ & OAJI (USA) & $=0.350$ \\
\hline
\end{tabular}

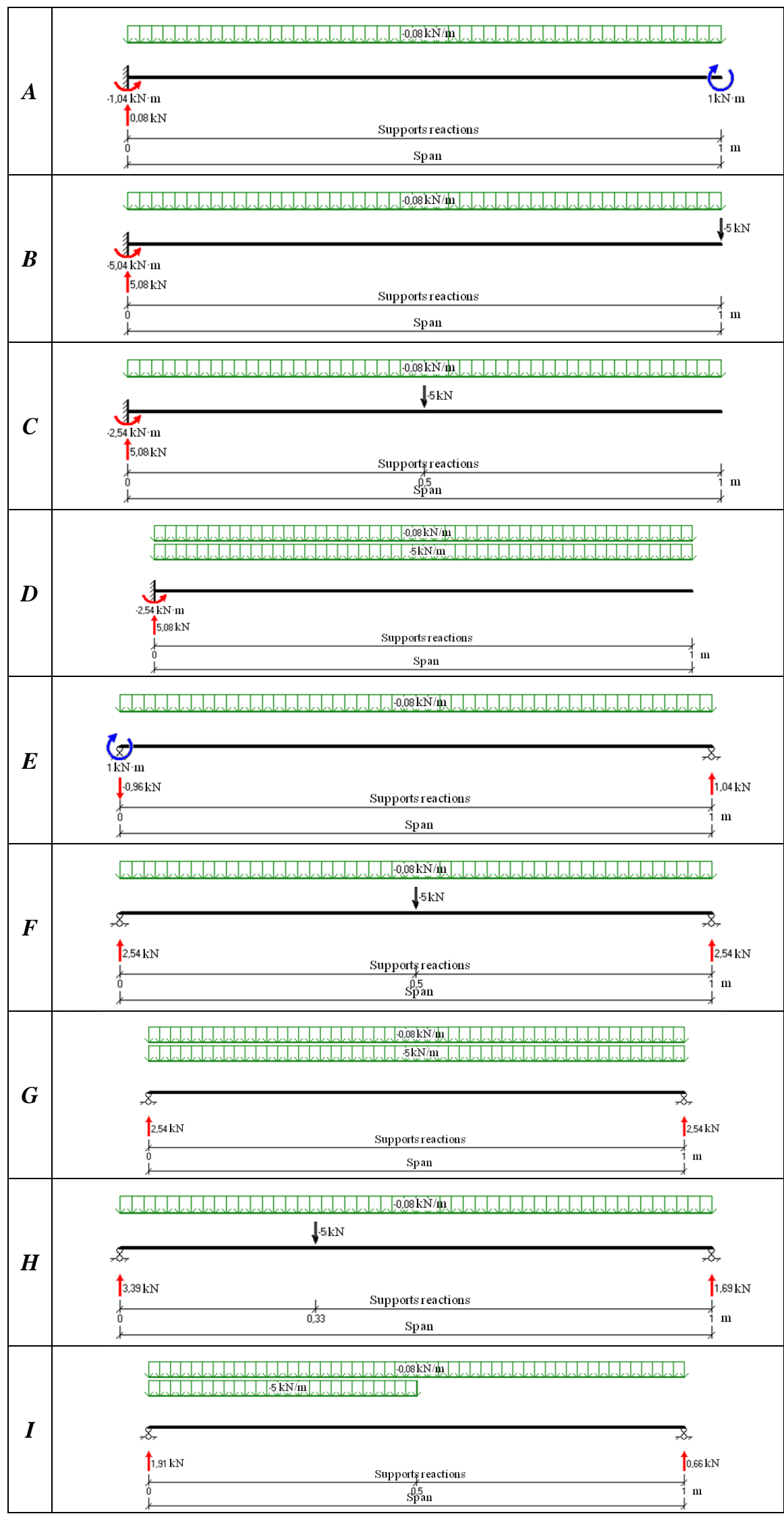

$A$ - the first scheme; $B$ - the second scheme; $C$ - the third scheme; $D$ - the fourth scheme; $E$ - the fifth scheme; $F$ - the sixth scheme; $G$ - the seventh scheme; $H$ - the eighth scheme; $I$ - the ninth scheme. 


\begin{tabular}{|c|c|c|c|c|c|c|}
\hline \multirow{4}{*}{ Impact Factor: } & ISRA (India) & $=3.117$ & SIS (USA) & $=0.912$ & ICV (Poland) & $=6.630$ \\
\hline & ISI (Dubai, UAE & $=0.829$ & РИНЦ (Russia) & 1) $=\mathbf{0 . 1 5 6}$ & PIF (India) & $=1.940$ \\
\hline & GIF (Australia) & $=0.564$ & ESJI (KZ) & $=8.716$ & IBI (India) & $=4.260$ \\
\hline & JIF & $=1.500$ & SJIF (Morocco & $=5.667$ & OAJI (USA) & $=0.350$ \\
\hline
\end{tabular}

$\boldsymbol{A}$

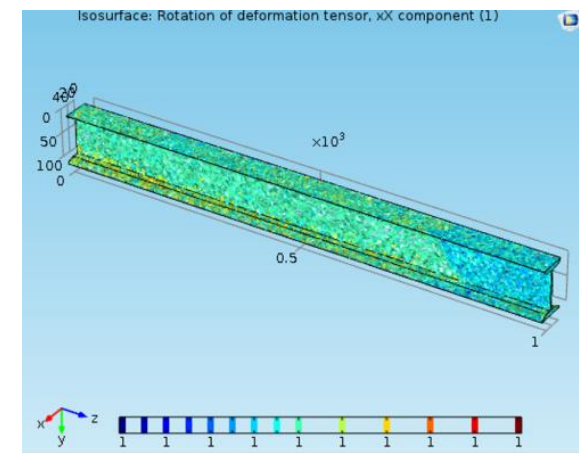

$C$
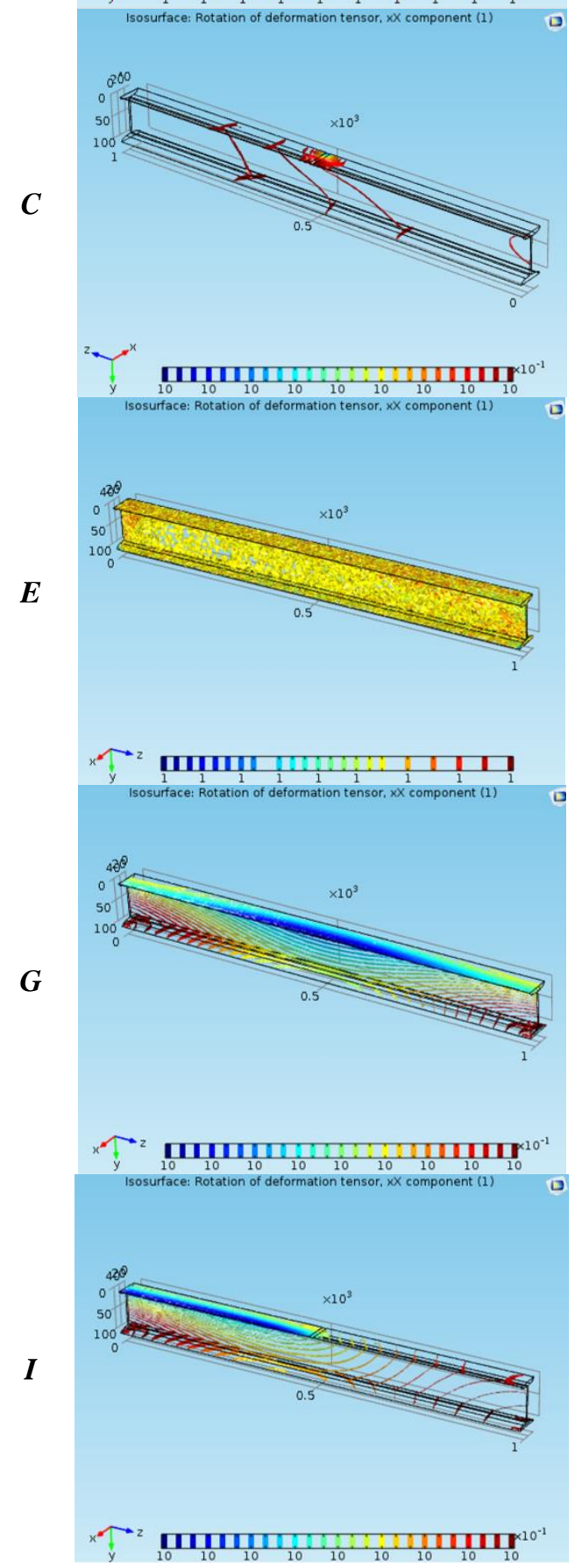

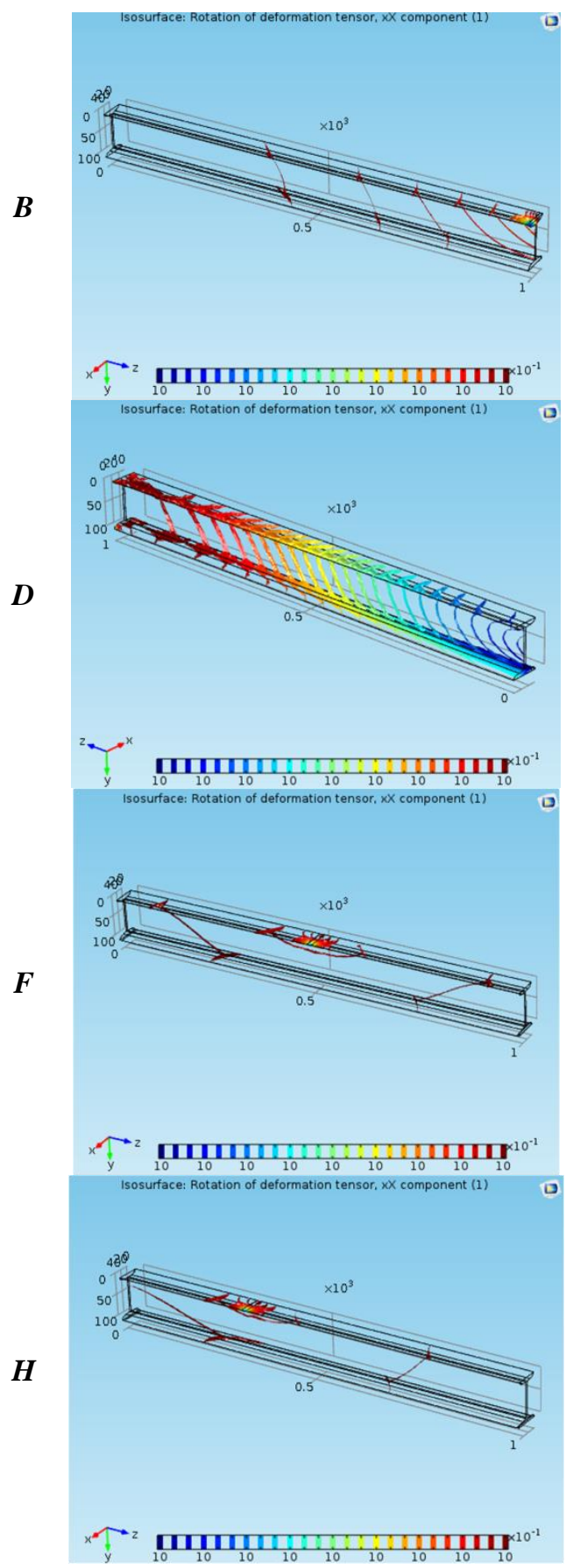

Figure 1 - Rotation of deformation tensor, $\boldsymbol{x} X$ component: $\boldsymbol{A}$ - the first scheme; $\boldsymbol{B}$ - the second scheme; $\boldsymbol{C}$ the third scheme; $D$ - the fourth scheme; $E$ - the fifth scheme; $F$ - the sixth scheme; $G$ - the seventh scheme; $H$ - the eighth scheme; $I$ - the ninth scheme. 


\begin{tabular}{|c|c|c|c|c|c|c|}
\hline \multirow{4}{*}{ Impact Factor: } & ISRA (India) & $=3.117$ & SIS (USA) & $=0.912$ & ICV (Poland) & $=6.630$ \\
\hline & ISI (Dubai, UAE & $=0.829$ & РИНЦ (Russia) & $=0.156$ & PIF (India) & $=1.940$ \\
\hline & GIF (Australia) & $=0.564$ & ESJI (KZ) & $=8.716$ & IBI (India) & $=4.260$ \\
\hline & JIF & $=1.500$ & SJIF (Morocco) & $=5.667$ & OAJI (USA) & $=0.350$ \\
\hline
\end{tabular}

$\boldsymbol{A}$

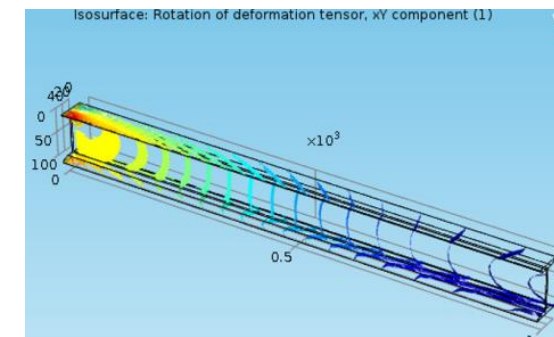

$C$
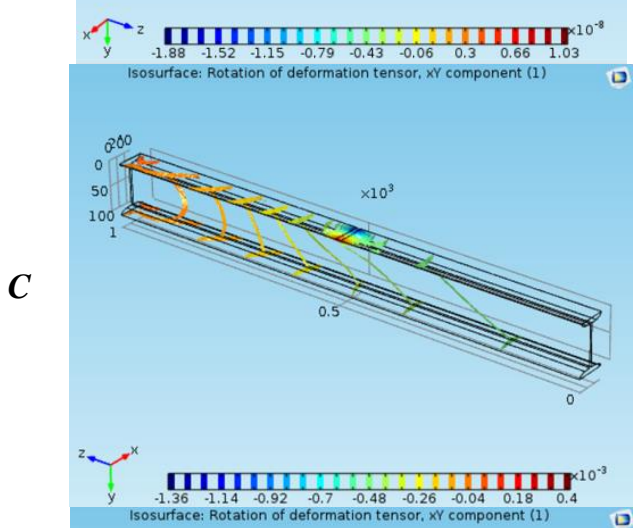

$\boldsymbol{E}$

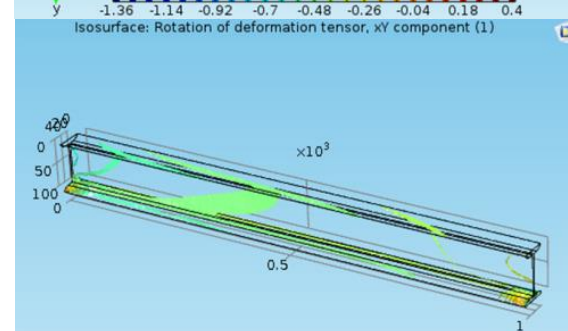

$\boldsymbol{G}$
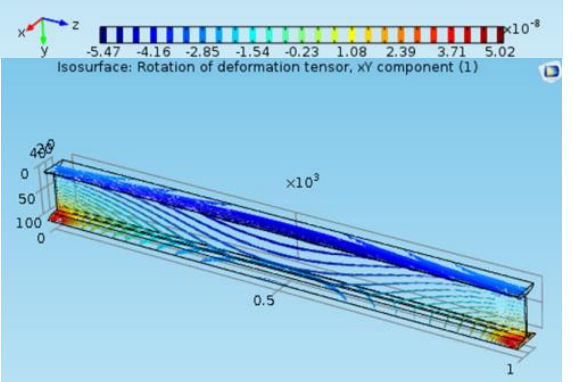

I

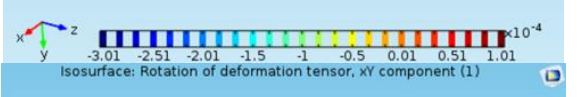

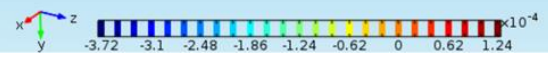

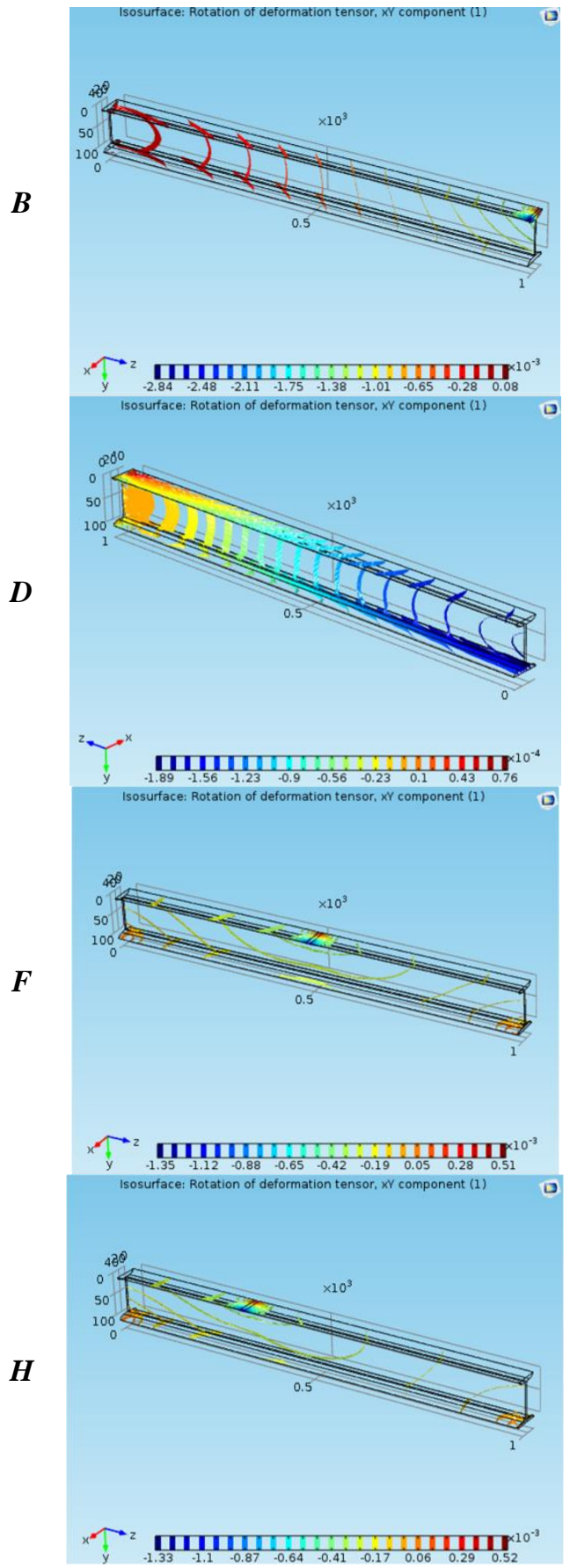

Figure 2 - Rotation of deformation tensor, $x Y$ component: $A$ - the first scheme; $B$ - the second scheme; $C$ the third scheme; $D$ - the fourth scheme; $E$ - the fifth scheme; $F$ - the sixth scheme; $G$ - the seventh scheme; $H$ - the eighth scheme; $I$ - the ninth scheme. 


\begin{tabular}{|c|c|c|c|c|c|c|}
\hline \multirow{4}{*}{ Impact Factor: } & ISRA (India) & $=3.117$ & SIS (USA) & $=0.912$ & ICV (Poland) & $=6.630$ \\
\hline & ISI (Dubai, UAE & $=0.829$ & РИНЦ (Russia) & 1) $=\mathbf{0 . 1 5 6}$ & PIF (India) & $=1.940$ \\
\hline & GIF (Australia) & $=0.564$ & ESJI (KZ) & $=8.716$ & IBI (India) & $=4.260$ \\
\hline & JIF & $=1.500$ & SJIF (Morocco & $=5.667$ & OAJI (USA) & $=0.350$ \\
\hline
\end{tabular}

$\boldsymbol{A}$

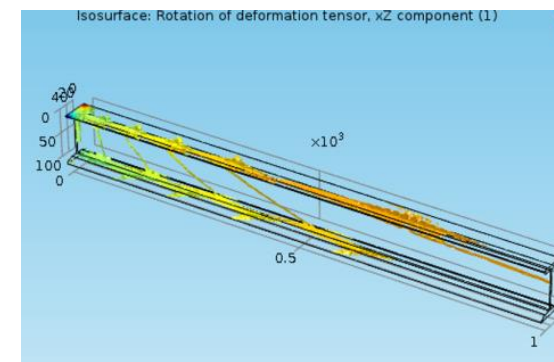

$C$
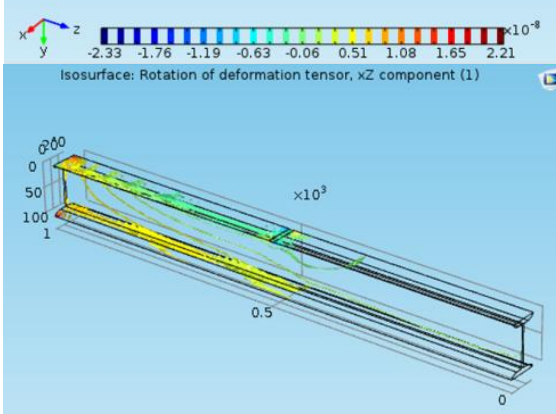

$\boldsymbol{E}$
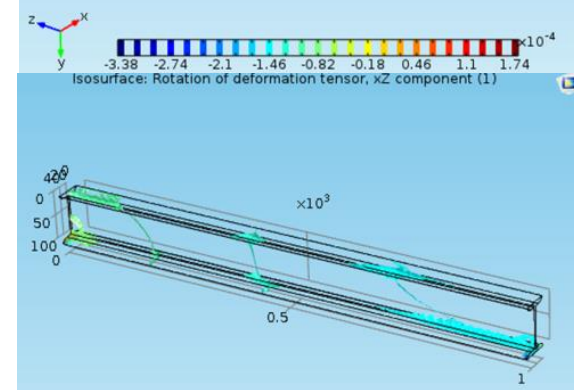

$\boldsymbol{G}$
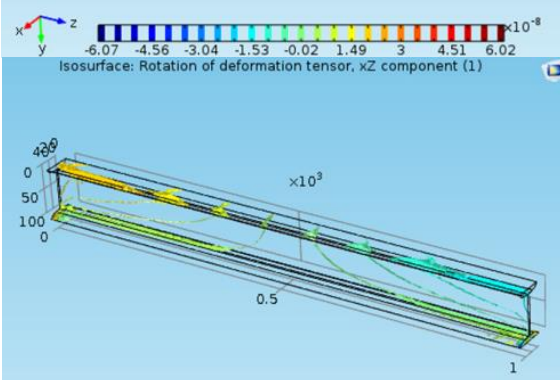

I

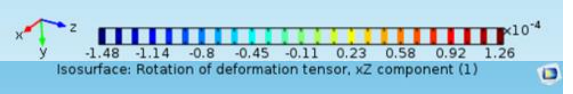

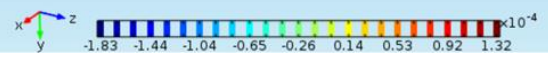

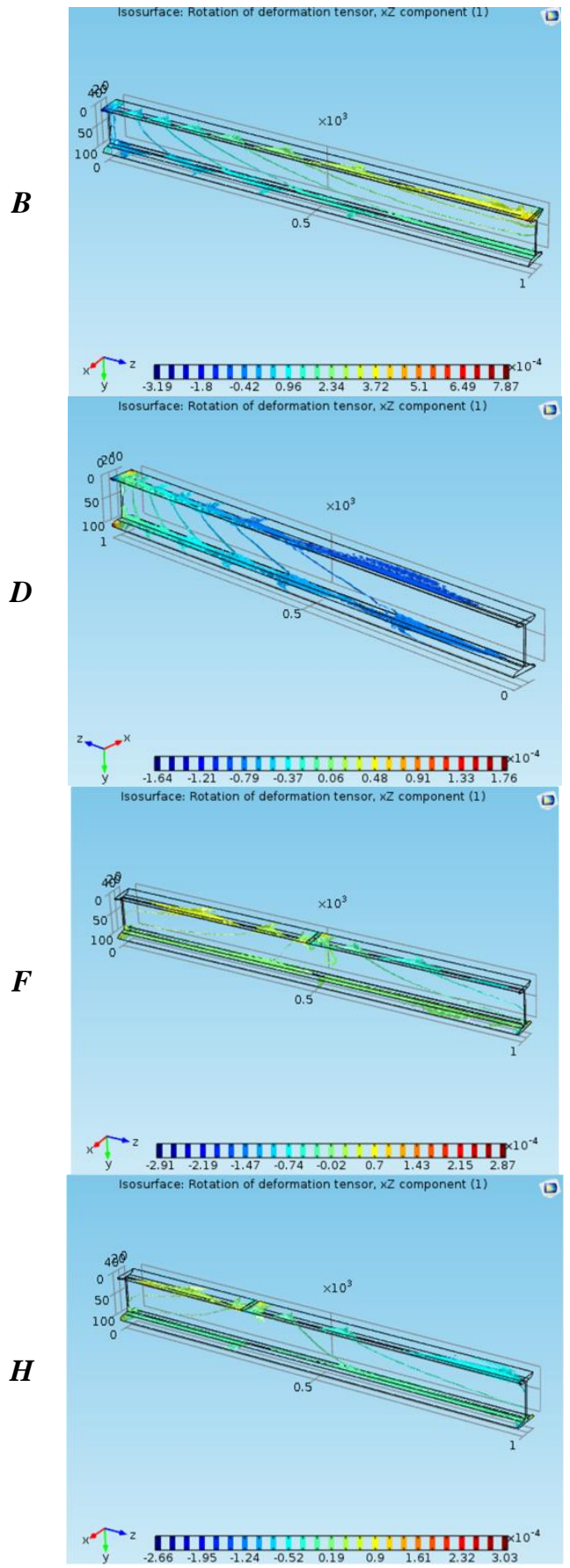

Figure 3 - Rotation of deformation tensor, $x Z$ component: $A$ - the first scheme; $B$ - the second scheme; $C$ the third scheme; $D$ - the fourth scheme; $E$ - the fifth scheme; $F$ - the sixth scheme; $G$ - the seventh scheme; $H$ - the eighth scheme; $I$ - the ninth scheme. 


\begin{tabular}{|c|c|c|c|c|c|c|}
\hline \multirow{4}{*}{ Impact Factor: } & ISRA (India) & $=3.117$ & SIS (USA) & $=0.912$ & ICV (Poland) & $=6.630$ \\
\hline & ISI (Dubai, UAE & $=0.829$ & РИНЦ (Russia) & 1) $=\mathbf{0 . 1 5 6}$ & PIF (India) & $=1.940$ \\
\hline & GIF (Australia) & $=0.564$ & ESJI (KZ) & $=8.716$ & IBI (India) & $=4.260$ \\
\hline & JIF & $=1.500$ & SJIF (Morocco & $=5.667$ & OAJI (USA) & $=0.350$ \\
\hline
\end{tabular}

$\boldsymbol{A}$

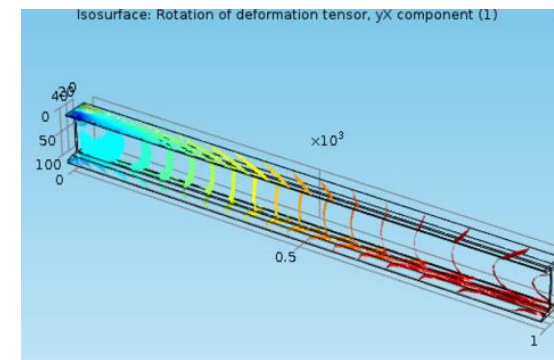

$C$
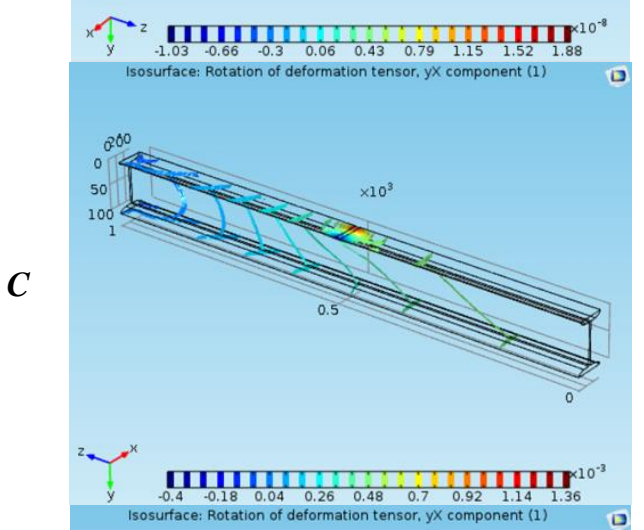

$\boldsymbol{E}$

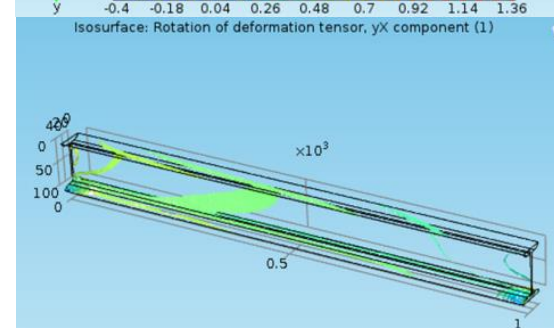

$\boldsymbol{G}$
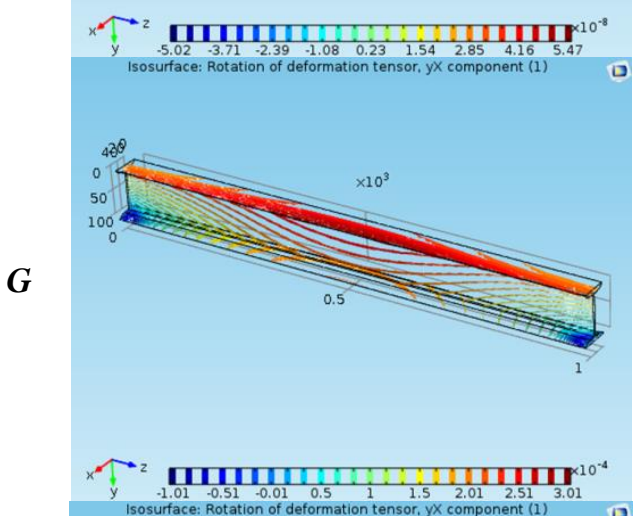

I

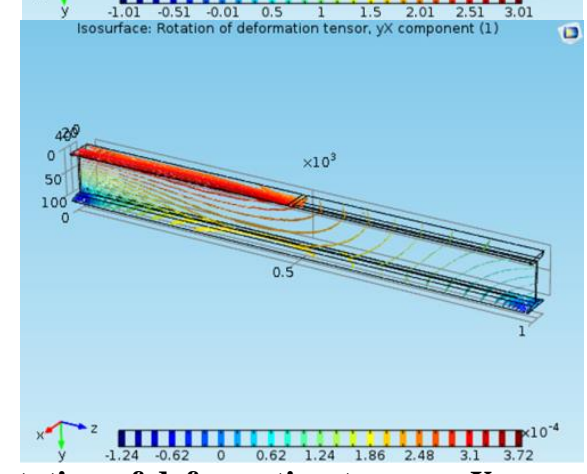

B

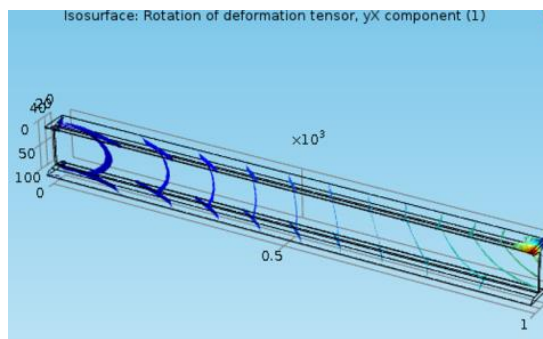

$D$
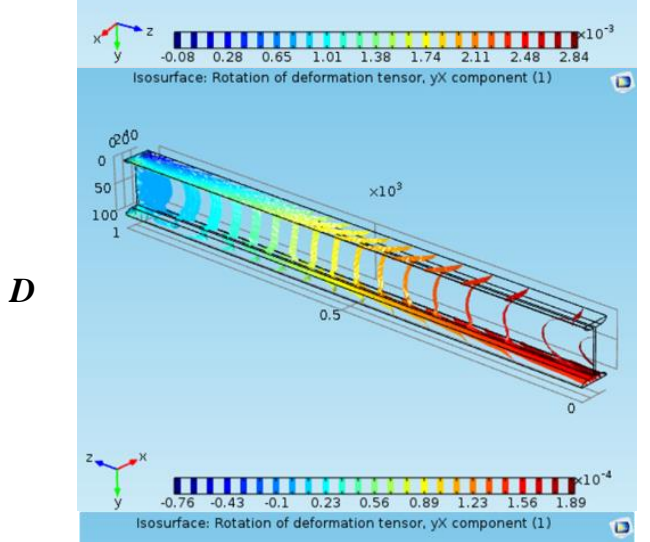

$\boldsymbol{F}$

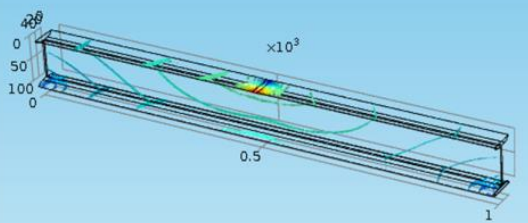

$\boldsymbol{H}$
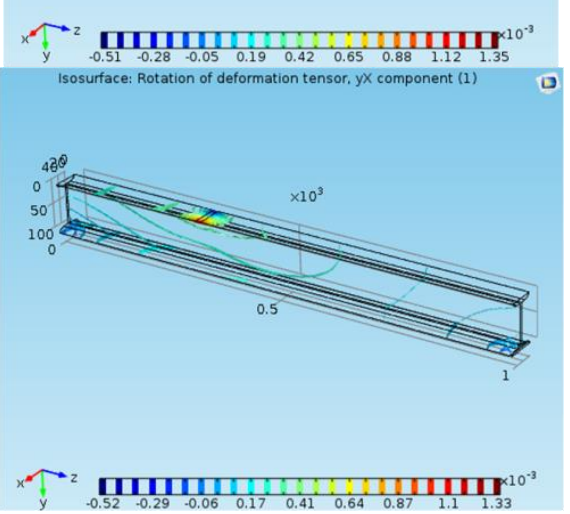

Figure 4 - Rotation of deformation tensor, $y X$ component: $A$ - the first scheme; $B$ - the second scheme; $C$ the third scheme; $D$ - the fourth scheme; $E$ - the fifth scheme; $F$ - the sixth scheme; $G$ - the seventh scheme; $H$ - the eighth scheme; $I$ - the ninth scheme. 


\begin{tabular}{|c|c|c|c|c|c|c|}
\hline \multirow{4}{*}{ Impact Factor: } & ISRA (India) & $=3.117$ & SIS (USA) & $=0.912$ & ICV (Poland) & $=6.630$ \\
\hline & ISI (Dubai, UAE & $=0.829$ & РИНЦ (Russia) & 1) $=\mathbf{0 . 1 5 6}$ & PIF (India) & $=1.940$ \\
\hline & GIF (Australia) & $=0.564$ & ESJI (KZ) & $=8.716$ & IBI (India) & $=4.260$ \\
\hline & JIF & $=1.500$ & SJIF (Morocco & $=5.667$ & OAJI (USA) & $=0.350$ \\
\hline
\end{tabular}

$\boldsymbol{A}$

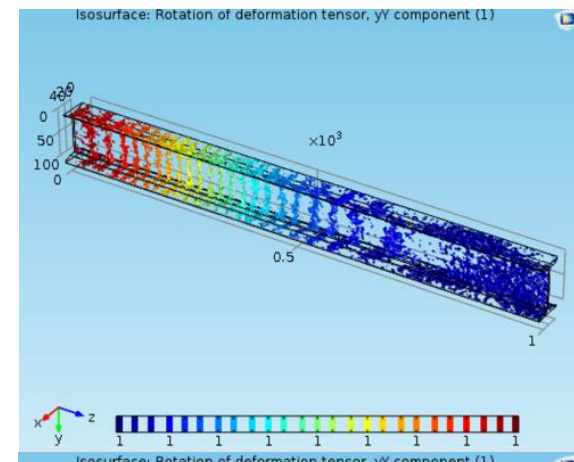

$C$
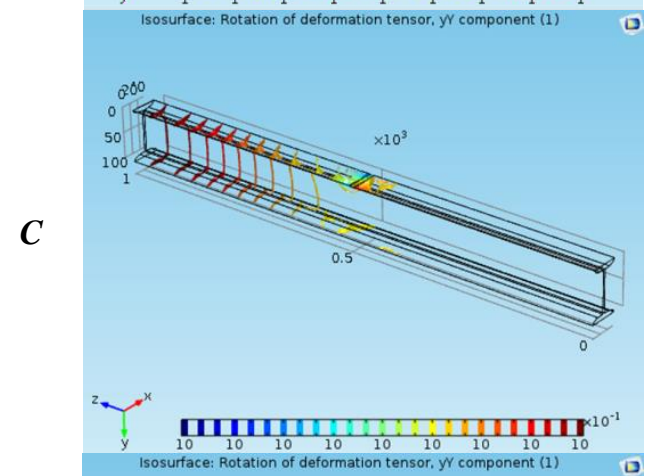

$\boldsymbol{E}$

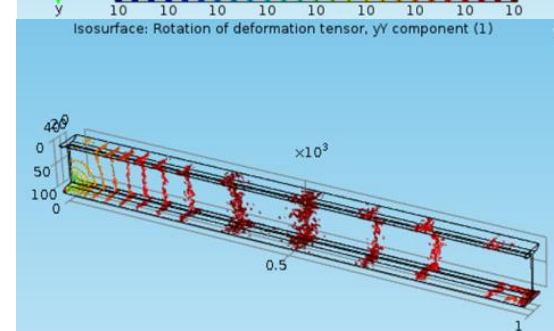

$\boldsymbol{G}$

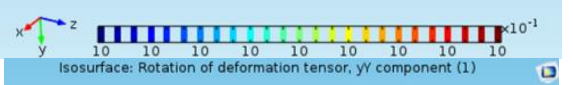

I

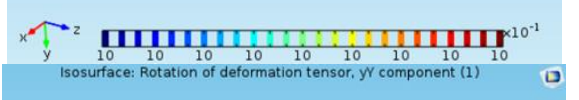

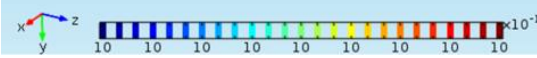

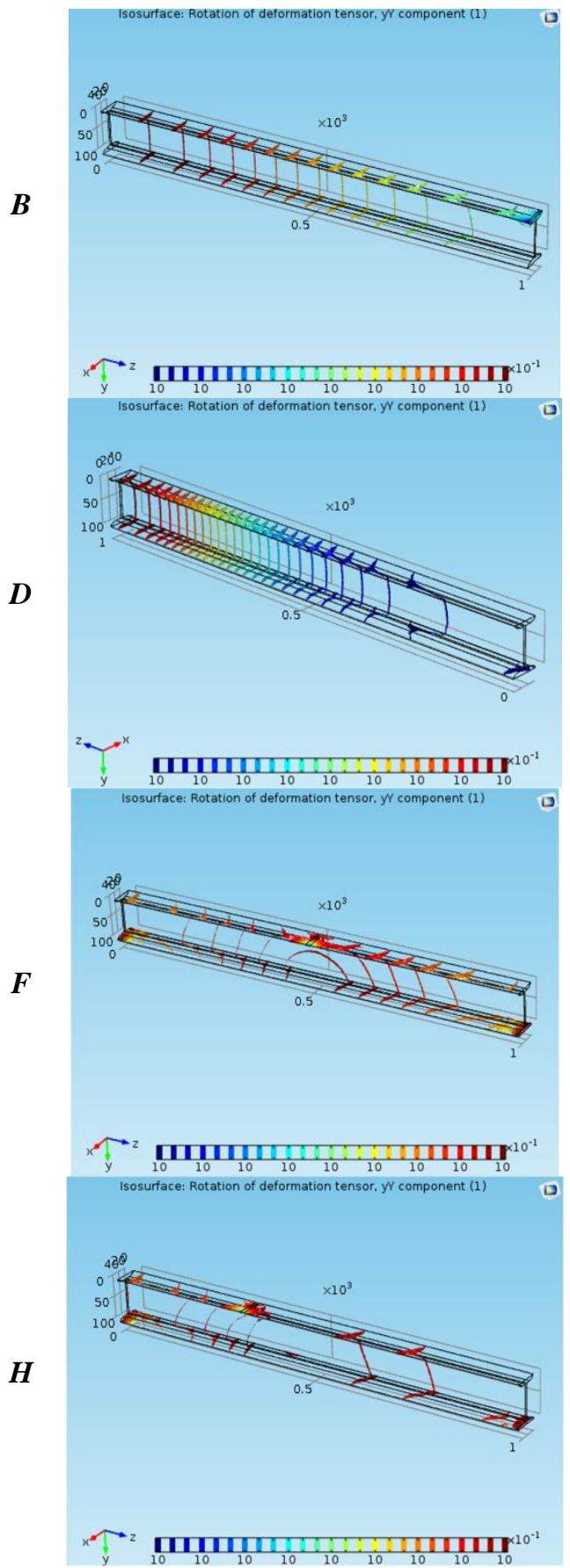

Figure 5 - Rotation of deformation tensor, $\boldsymbol{y} Y$ component: $\boldsymbol{A}$ - the first scheme; $\boldsymbol{B}$ - the second scheme; $\boldsymbol{C}$ the third scheme; $D$ - the fourth scheme; $E$ - the fifth scheme; $F$ - the sixth scheme; $G$ - the seventh scheme; $H$ - the eighth scheme; $I$ - the ninth scheme. 


\begin{tabular}{|c|c|c|c|c|c|c|}
\hline \multirow{4}{*}{ Impact Factor: } & ISRA (India) & $=3.117$ & SIS (USA) & $=0.912$ & ICV (Poland) & $=6.630$ \\
\hline & ISI (Dubai, UAE & $=0.829$ & РИНЦ (Russia) & 1) $=\mathbf{0 . 1 5 6}$ & PIF (India) & $=1.940$ \\
\hline & GIF (Australia) & $=0.564$ & ESJI (KZ) & $=8.716$ & IBI (India) & $=4.260$ \\
\hline & JIF & $=1.500$ & SJIF (Morocco & $=5.667$ & OAJI (USA) & $=0.350$ \\
\hline
\end{tabular}

A

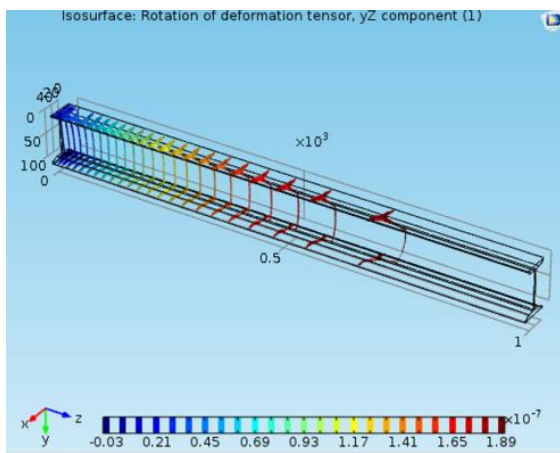

$C$

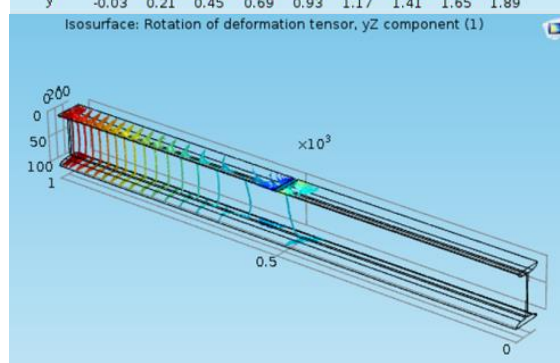

$\boldsymbol{E}$

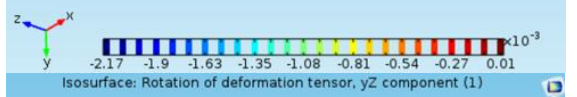

$\boldsymbol{G}$

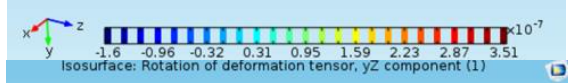

$\boldsymbol{I}$
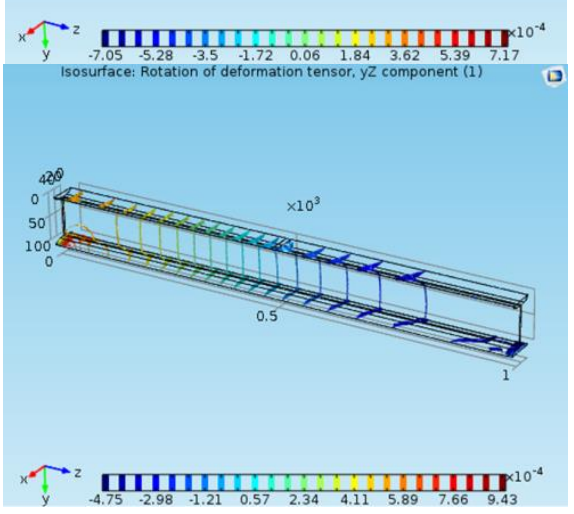

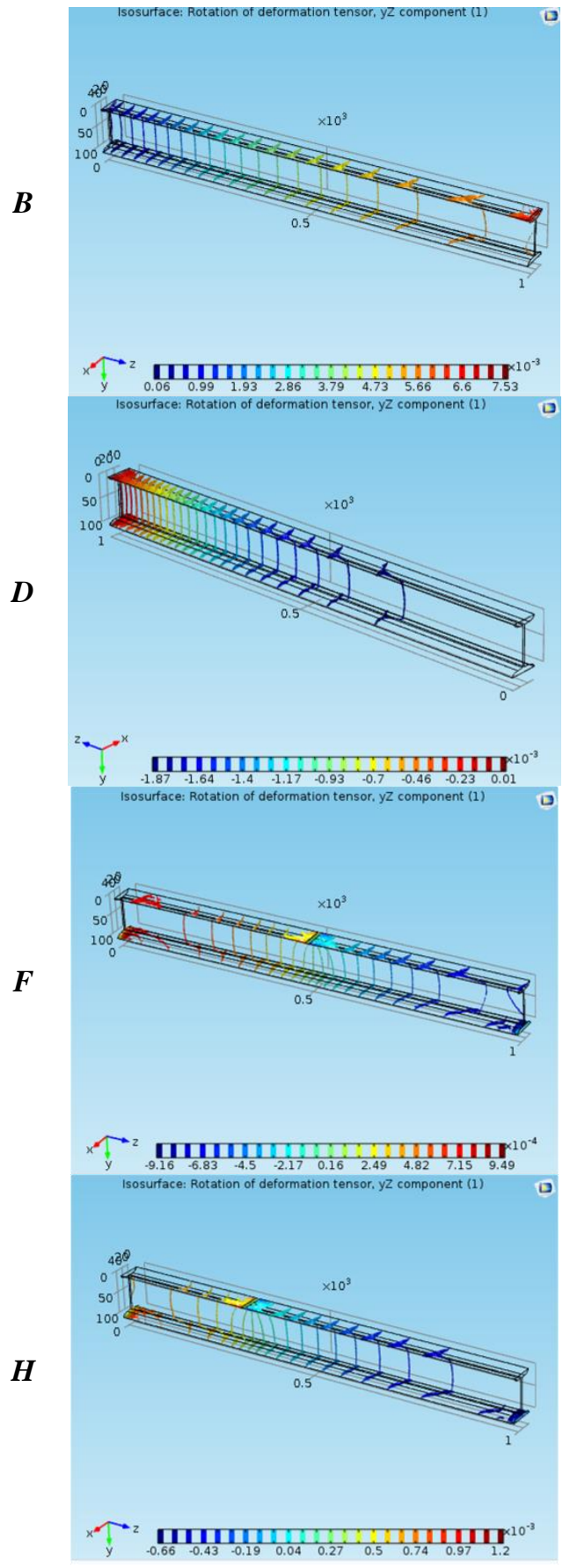

Figure 6 - Rotation of deformation tensor, $y Z$ component: $A$ - the first scheme; $B$ - the second scheme; $C$ the third scheme; $D$ - the fourth scheme; $E$ - the fifth scheme; $F$ - the sixth scheme; $G$ - the seventh scheme; $H$ - the eighth scheme; $I$ - the ninth scheme. 


\begin{tabular}{|c|c|c|c|c|c|c|}
\hline \multirow{4}{*}{ Impact Factor: } & ISRA (India) & $=3.117$ & SIS (USA) & $=0.912$ & ICV (Poland) & $=6.630$ \\
\hline & ISI (Dubai, UAE & $=0.829$ & РИНЦ (Russia) & 1) $=\mathbf{0 . 1 5 6}$ & PIF (India) & $=1.940$ \\
\hline & GIF (Australia) & $=0.564$ & ESJI (KZ) & $=8.716$ & IBI (India) & $=4.260$ \\
\hline & JIF & $=1.500$ & SJIF (Morocco & $=5.667$ & OAJI (USA) & $=0.350$ \\
\hline
\end{tabular}

$\boldsymbol{A}$

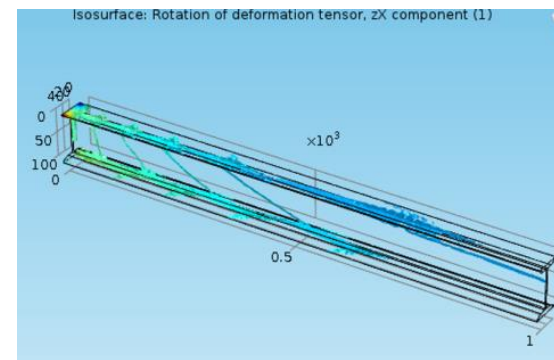

$C$
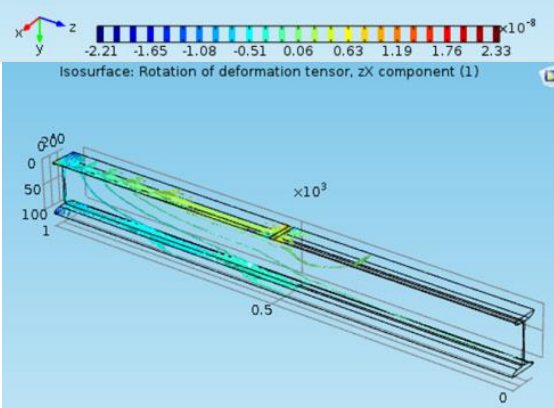

$\boldsymbol{E}$

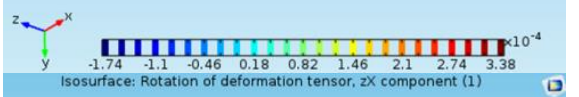

$\boldsymbol{G}$
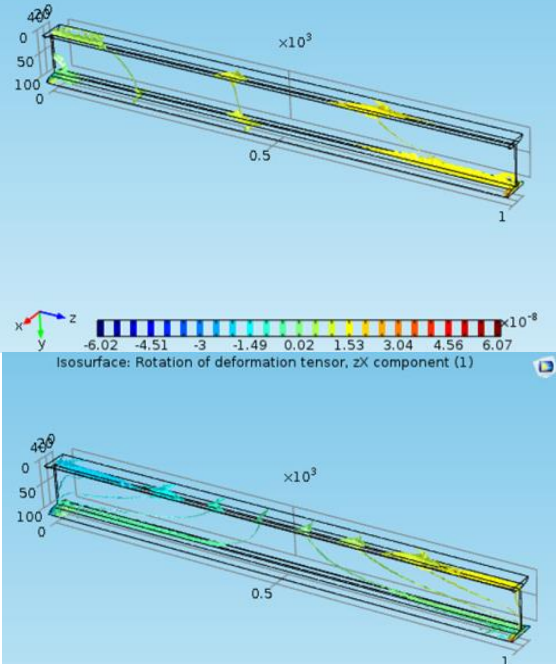

I

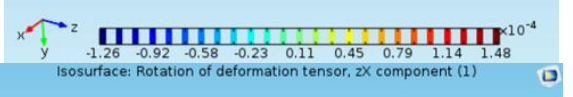

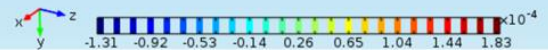

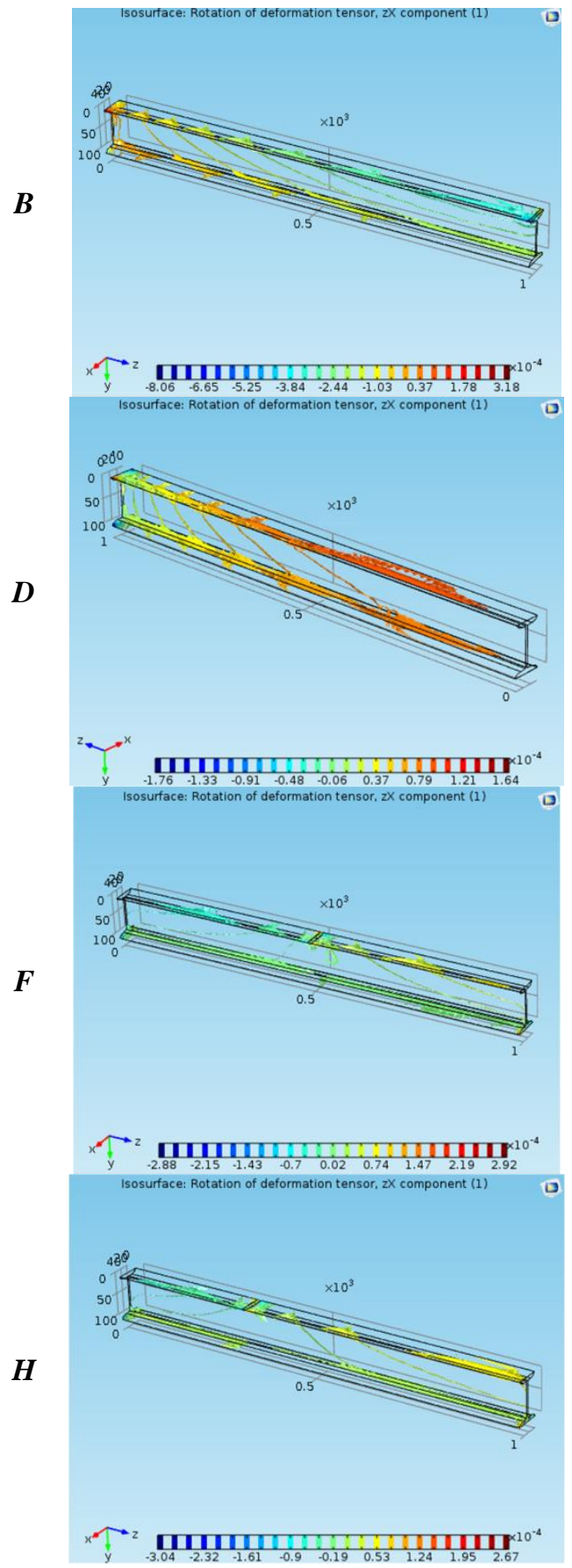

Figure 7 - Rotation of deformation tensor, $z X$ component: $A$ - the first scheme; $B$ - the second scheme; $C$ the third scheme; $D$ - the fourth scheme; $E$ - the fifth scheme; $F$ - the sixth scheme; $G$ - the seventh scheme; $H$ - the eighth scheme; $I$ - the ninth scheme. 


\begin{tabular}{|c|c|c|c|c|c|c|}
\hline \multirow{4}{*}{ Impact Factor: } & ISRA (India) & $=3.117$ & SIS (USA) & $=0.912$ & ICV (Poland) & $=6.630$ \\
\hline & ISI (Dubai, UAE & $=0.829$ & РИНЦ (Russia) & 1) $=\mathbf{0 . 1 5 6}$ & PIF (India) & $=1.940$ \\
\hline & GIF (Australia) & $=0.564$ & ESJI (KZ) & $=8.716$ & IBI (India) & $=4.260$ \\
\hline & JIF & $=1.500$ & SJIF (Morocco & $=5.667$ & OAJI (USA) & $=0.350$ \\
\hline
\end{tabular}

$\boldsymbol{A}$

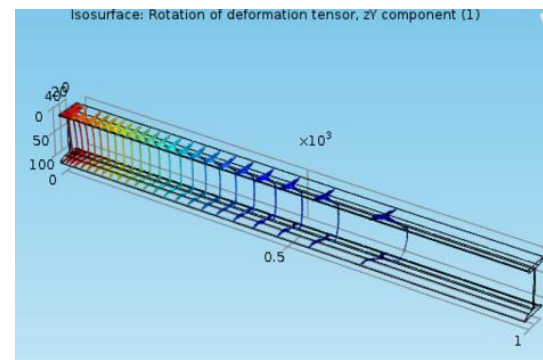

$C$
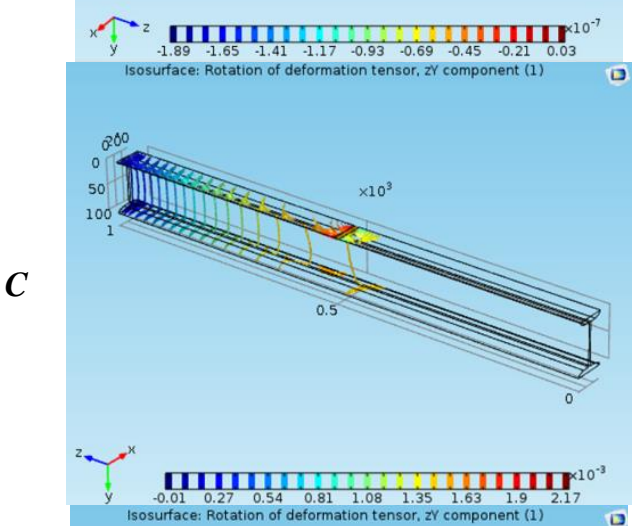

$\boldsymbol{E}$

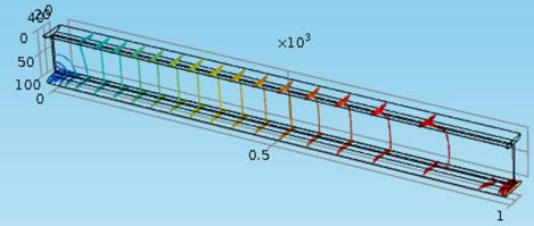

$\boldsymbol{G}$
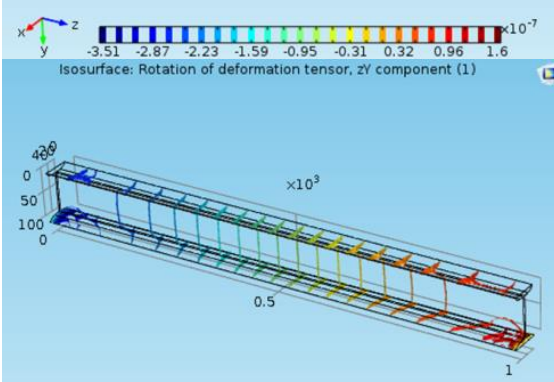

$I$

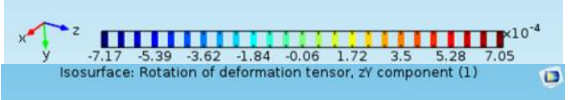

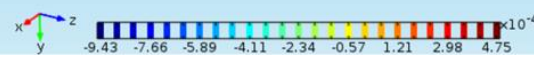

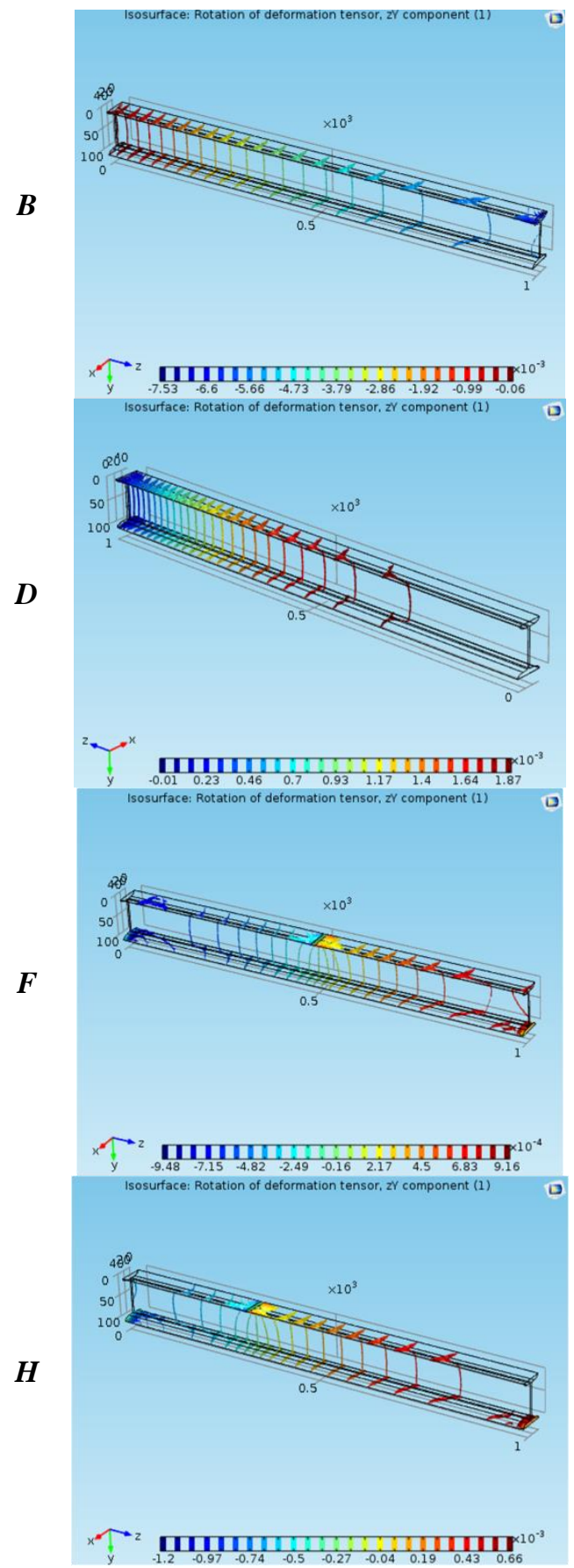

Figure 8 - Rotation of deformation tensor, $z Y$ component: $A$ - the first scheme; $B$ - the second scheme; $C$ the third scheme; $D$ - the fourth scheme; $E$ - the fifth scheme; $F$ - the sixth scheme; $G$ - the seventh scheme; $H$ - the eighth scheme; $I$ - the ninth scheme. 


\begin{tabular}{|c|c|c|c|c|c|c|}
\hline \multirow{4}{*}{ Impact Factor: } & ISRA (India) & $=3.117$ & SIS (USA) & $=0.912$ & ICV (Poland) & $=6.630$ \\
\hline & ISI (Dubai, UAE & $=0.829$ & РИНЦ (Russia) & 1) $=\mathbf{0 . 1 5 6}$ & PIF (India) & $=1.940$ \\
\hline & GIF (Australia) & $=0.564$ & ESJI (KZ) & $=8.716$ & IBI (India) & $=4.260$ \\
\hline & JIF & $=1.500$ & SJIF (Morocco & $=5.667$ & OAJI (USA) & $=0.350$ \\
\hline
\end{tabular}

$\boldsymbol{A}$

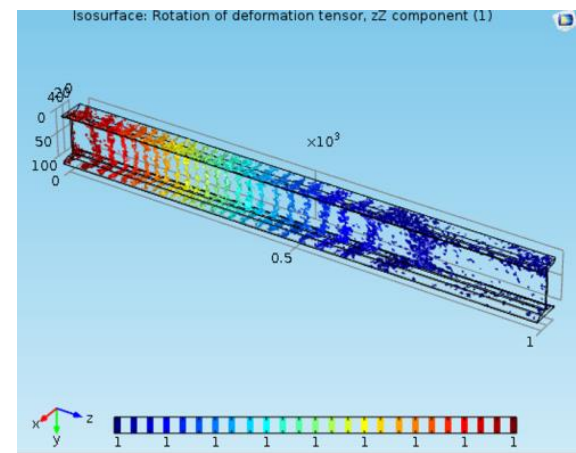

$C$

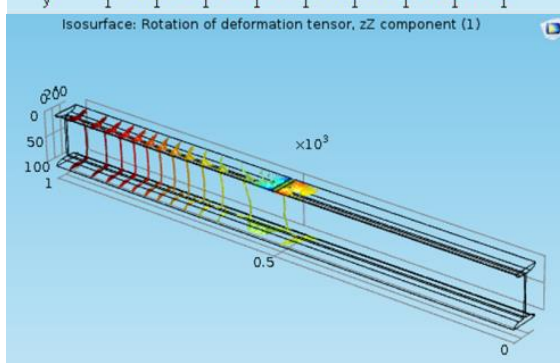

$\boldsymbol{E}$

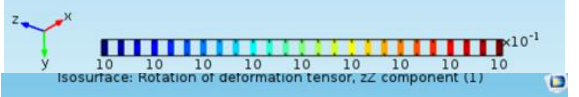

$\boldsymbol{G}$
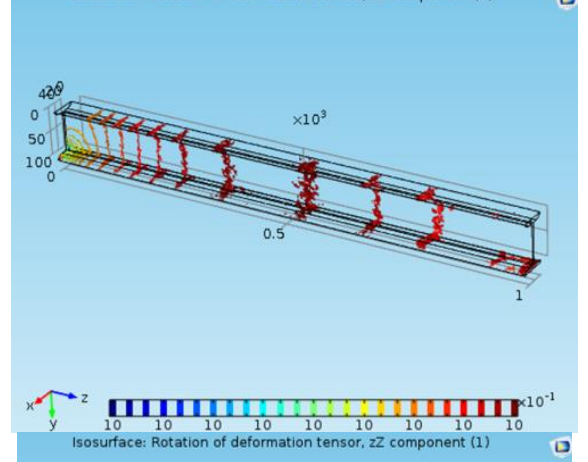

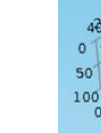
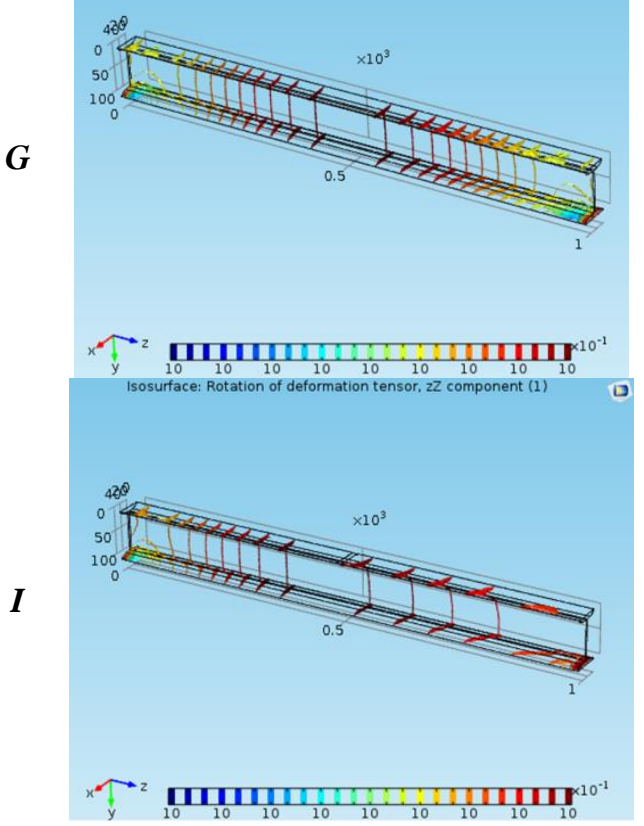

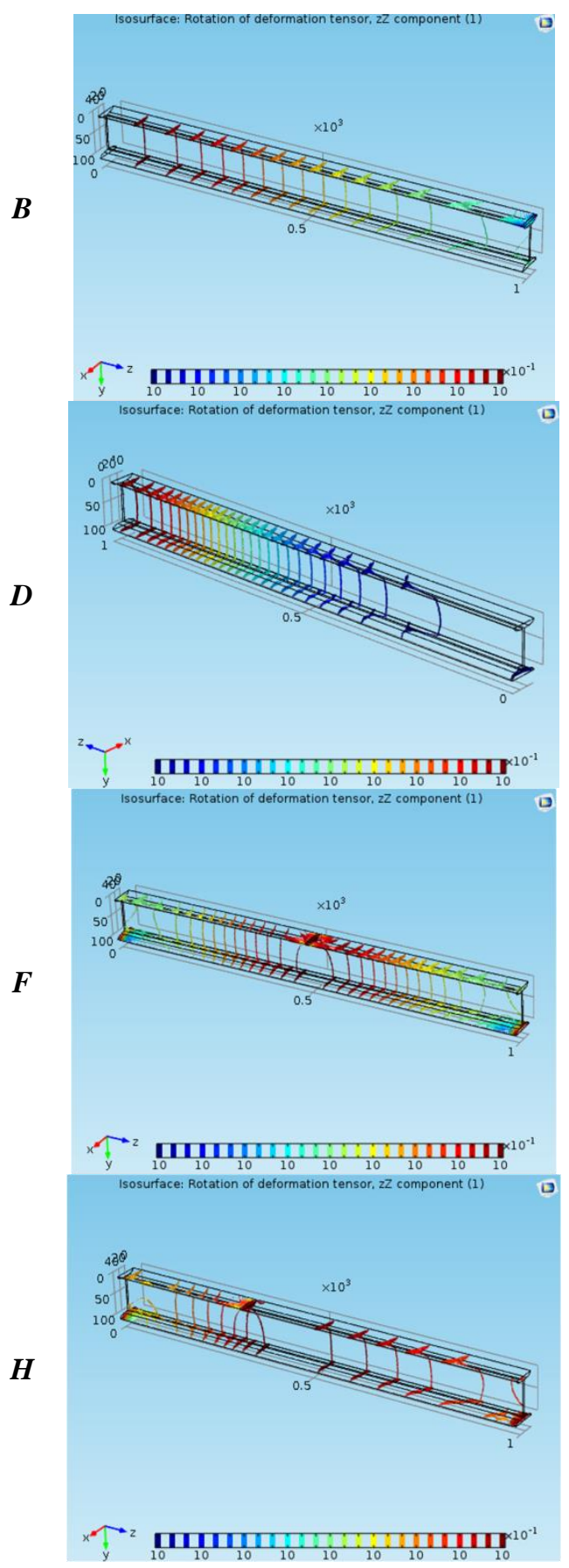

Figure 9 - Rotation of deformation tensor, $z Z$ component: $A$ - the first scheme; $B$ - the second scheme; $C$ the third scheme; $D$ - the fourth scheme; $E$ - the fifth scheme; $F$ - the sixth scheme; $G$ - the seventh scheme; $H$ - the eighth scheme; $I$ - the ninth scheme. 


\begin{tabular}{|c|c|c|c|c|c|c|}
\hline \multirow{4}{*}{ Impact Factor: } & ISRA (India) & $=\mathbf{3 . 1 1 7}$ & SIS (USA) & $=0.912$ & ICV (Poland) & $=6.630$ \\
\hline & ISI (Dubai, UAE & $=0.829$ & РИНЦ (Russia & $=0.156$ & PIF (India) & $=1.940$ \\
\hline & GIF (Australia) & $=0.564$ & ESJI (KZ) & $=8.716$ & IBI (India) & $=4.260$ \\
\hline & JIF & $=1.500$ & SJIF (Morocco & $=5.667$ & OAJI (USA) & $=0.350$ \\
\hline
\end{tabular}

Table 2. Maximum deflections of the I-beams.

\begin{tabular}{|c|c|c|c|c|c|c|c|c|c|}
\hline The scheme number & 1st & 2nd & 3rd & 4th & 5th & 6th & 7th & 8th & 9th \\
\hline Maximum deflection, mm & 1.74 & 5.71 & 1.81 & 2.16 & 0.22 & 0.36 & 0.23 & 0.31 & 0.12 \\
\hline
\end{tabular}

\section{References:}

1. Wang, Y. Q., et al. (2011). Experimental Study on the Deformation Behavior of Symmetric Stainless Steel I-Beams. Applied Mechanics and Materials, Vol. 94-96, 18-24.

2. Chemezov, D., Osipov, T., \& Pesenko, A. (2016). A static calculation of an I-beam. ISJ Theoretical \& Applied Science, 11 (43), 49-52.

3. Chemezov, D., et al. (2019). Bendings of cantilever and doubly supported steel I-beams. ISJ Theoretical \& Applied Science, 01 (69), 261267.

4. Chemezov, D., et al. (2019). Isosurfaces of mechanical stresses in cantilever and doubly supported steel I-beams subject to bending. ISJ Theoretical \& Applied Science, 02 (70), 301339.

5. Gao, B. (2011). Research on Nonlinear Deformation and Stability of Stainless Steel Flexural Members with Welded I-Sections. Dissertation of Chongqing University, in Chinese.
6. Wang, C. M., Reddy, J. N. \& Lee, K. H. (2000). Shear Deformable Beams and Plates. 1st Edition, $312 \mathrm{p}$.

7. Holodnyak, Y. S., Perig, A. V., \& Matveev, I. A. (2012). I-bar strength computation methodology perfection for teaching of engineering disciplines. PNRPU Mechanics Bulletin, 4(14), 77-90.

8. Kuznetsov, I. L., Aktuganov, A. A., \& Trofimov, A. P. (2010). Designing and research of a composite steel and wood (flitched) I-beam. News of the Kazan State University of Architecture and Engineering, 1(13), 117-121.

9. Tuysina, E. B., \& Kaymov, R. A. (2014). Calculation of the I-beam under the action of irregular cyclic loading. News of the Kazan State University of Architecture and Engineering, 4(30), 335-340.

10. Limantsev, A. A., \& Denisenko, A. V. (2018). Determination of optimal design resistance and section shape of bilateral beam. International research journal, 01(67), 110-115. 